\title{
PREÇO INTERNACIONAL, TAXA DE CÂMBIO E O PREÇO PAGO AOS PRODUTORES BRASILEIROS DE SOJA ${ }^{1}$
}

\author{
Lucas Oliveira de Sousa ${ }^{2}$ \\ Marcelo Dias Paes Ferreira ${ }^{3}$
}

Resumo: O objetivo deste estudo foi verificar como o preço internacional da soja e a taxa de câmbio influenciaram a formação dos preços domésticos da soja nos estados de Mato Grosso, Paraná, Rio Grande do Sul e Goiás, os maiores produtores dessa commodity no Brasil, de 1996 a 2011. Para isso, foram utilizados o modelo de correção de erro vetorial (VEC) e o Teste de Causalidade de Granger. Os resultados indicaram que variações no preço internacional da soja e na taxa de câmbio afetaram os preços da soja nos quatro estados e que Mato Grosso foi o único cujo preço não sofreu influência dos demais estados. Variações no mercado internacional de soja e no mercado de câmbio são repassadas integralmente aos preços domésticos no longo prazo, sendo estes preços mais influenciados pelo preço internacional. No curto prazo, os mercados seguiram a relação de preço de Mato Grosso com o preço internacional e a taxa de câmbio. Conclui-se que a preocupação dos produtores com variações na taxa de câmbio é pertinente, porém o preço internacional é o principal elemento da formação dos preços domésticos, que os mercados analisados são integrados e que Mato Grosso apresenta relevância na formação dos preços nos principais mercados dessa commodity.

Palavras-chave: Brasil, estados, preço internacional, taxa de câmbio

1 Recebido em: 06/02/2014; Aceito em: 20/05/2014.

2 Mestre em Economia Aplicada pela Universidade Federal de Viçosa. Professor do Departamento de Zootecnia e Extensão Rural - Faculdade de Agronomia, Medicina Veterinária e Zootecnia - Universidade Federal de Mato Grosso. E-mail: lucas.agronegocio@gmail.com.

3 Mestre em Economia Aplicada pela Universidade Federal de Viçosa. Doutorando do Programa de PósGraduação em Economia Aplicada - Departamento de Economia Rural - Universidade Federal de Viçosa. E-mail: marcelo_dpf@yahoo.com.br. 
Abstract: This paper aimed to verify how the international soybean price and the exchange rate influenced the formation of domestic soybean prices in the four largest producers of this commodity in Brazil - Mato Grosso, Paraná, Rio Grande do Sul and Goiás, from 1996 to 2011. For this, the vector error correction (VEC) model and the Test of Granger Causality were used. The results indicated that variations in international soybean price and in the exchange rate affect soybean prices in the four states, and Mato Grosso was the only state whose price was not influenced by the other states prices. In the long run, changes in the international soybean market and the foreign exchange market are fully transmitted to domestic prices and these prices are more influenced by the international price. In the short run, the markets followed the price relation of Mato Grosso with the international price and exchange rate. We conclude that the concern of producers about changes in the exchange rate is relevant, but the international price is the main element of the formation of domestic prices, the markets analyzed are integrated and Mato Grosso has relevance in pricing in key markets of this commodity.

Keywords: Brazil, exchange rate, international price, states

\section{Introdução}

Há um debate no setor agrícola acerca do impacto de mudanças cambiais sobre a renda dos produtores rurais. Essa relação é mais enfatizada no caso de culturas cujos preços domésticos dependem das cotações internacionais, definidas em bolsas de mercadoria no exterior, caso da soja, cujo complexo agroindustrial é o principal gerador de divisas cambiais do Brasil (BRASIL, 2013a).

O Brasil é o segundo maior produtor e exportador mundial de soja, ficando atrás apenas dos Estados Unidos da América. Internamente, a produção concentra-se nas regiões Centro-Oeste e Sul. Em 2011, do total de 74,82 milhões de toneladas, $74,41 \%$ foram originadas dos estados de Mato Grosso (27,8\%), Paraná (20,7\%), Rio Grande do Sul (15,7\%) e Goiás $(10,3 \%)$ (IBGE, 2013). As exportações seguem esse mesmo padrão. O Brasil exportou cerca de 32,9 milhões de toneladas de soja ${ }^{4}$ em 2011 e 2012, sendo que $75 \%$ desse total, em 2011, e $72 \%$ em 2012, foram exportados pelos quatro estados citados anteriormente (BRASIL, 2013b).

4 Cesta de Produtos (NCM): 12010010, 12010090, 12011000, 12019000. 
O preço internacional da soja é definido na Chicago Board of Trade (CBOT), nos Estados Unidos da América e, por conseguinte, cotado em dólar (US\$). Logo, o preço pago ao produtor brasileiro depende da cotação internacional da soja, da taxa de câmbio (R\$/US\$), além de fatores como frete, custos de armazenagem, políticas setoriais, entre outros, que fazem com que o preço varie entre os estados produtores.

No passado, até o final da década de 1980, quando parte dos mercados de commodities agropecuárias eram regulamentados por acordos internacionais e, ou por políticas governamentais de preço, buscava-se verificar a existência ou não de transmissão dos preços internacionais para os preços domésticos, com pouca ênfase nos efeitos da taxa de câmbio (MUNDLAK; LARSON, 1992). Atualmente, diante do cenário de desregulamentação de preços, a dinâmica de formação de preços foi afetada e, portanto, ressalta-se a relevância de análises da influência de variações cambiais sobre o preço doméstico de commodities.

Vários estudos têm abordado a temática de integração entre o mercado nacional e internacional de commodities agropecuárias. Mundlak e Larson (1992) atestaram a existência de transmissão de preço e verificaram que o preço internacional era o principal componente das variações nos preços domésticos de uma série de produtos analisados em diversos países. Baffes e Gardner (2003) analisaram o grau de transmissão de preços internacionais de dez commodities agrícolas para os preços domésticos dessas commodities em oitos países em desenvolvimento e verificaram que apenas três desses mercados eram integrados ao mercado mundial.

Outros trabalhos têm dado ênfase à dinâmica de preços no mercado brasileiro. Margarido et al. (2007) verificaram que, no longo prazo, variações no preço internacional do trigo e na taxa de câmbio são plenamente transmitidas para o preço da farinha de trigo em São Paulo, tendo o efeito preço prevalecido sobre o efeito câmbio. Margarido, Turolla e Bueno (2007) analisaram a transmissão de preços no mercado mundial de soja e atestaram que, no longo prazo, o preço internacional é plenamente transmitido para o preço doméstico da soja no Brasil, que é considerado um tomador de preço. Ao analisarem como as variações 
na taxa de câmbio eram transmitidas para os preços de exportação da soja no Brasil, Fraga et al. (2008) verificaram que a política cambial pode ocasionar impactos positivos sobre o volume exportado, mas não pode ser a única política de estímulo. Mendonça et al. (2011) verificaram a existência de integração entre os preços dos principais estados brasileiros produtores de soja, sem, contudo, considerar os efeitos do preço internacional e da taxa de câmbio, por se tratar de uma análise de integração regional dentro do país. O presente trabalho complementa os estudos anteriores no sentido de medir a influência do preço internacional e da taxa de câmbio sobre o preço pago aos produtores brasileiros.

Diante do exposto, o objetivo deste estudo foi verificar como o preço internacional da soja e a taxa de câmbio têm influenciado a formação do preço doméstico da soja nos estados de Mato Grosso, Paraná, Rio Grande do Sul e Goiás, os maiores produtores dessa commodity no Brasil, de 1996 a 2011.

Os resultados deste trabalho auxiliariam na tomada de decisão dos produtores de soja, das cooperativas, da indústria de processamento e dos demais agentes que atuam no setor, permitindo aos formuladores de políticas para o setor, principalmente o Governo Federal, entender como os choques oriundos do mercado internacional e do mercado de câmbio afetariam os preços no mercado nacional de soja. Além desta introdução, o trabalho é composto pelas seguintes seções: metodologia, resultados e discussão, considerações finais e referências.

\section{Metodologia}

\subsection{Referencial Teórico}

A fundamentação teórica para trabalhos que analisam a integração entre mercados nacional e internacional é a lei do preço único. Segundo 
Krugman e Obstfeld (2005), esta lei postula que bens idênticos vendidos em países diferentes devem ser vendidos pelo mesmo preço quando expressos na mesma moeda, condicionado à concorrência perfeita dos mercados, à ausência de custos de transporte e à não incidência de barreiras ao comércio.

Segundo Margarido, Fernandes e Turolla (2002), a validade da lei do preço único relaciona-se diretamente ao processo de arbitragem internacional, o qual, no longo prazo, induz a convergência dos preços nos mercados doméstico e externo a um mesmo valor. Logo, caso a lei do preço único seja válida, verificar-se-á a transmissão integral de preços, e o comportamento do preço doméstico de determinada commodity seguirá os movimentos do preço externo dessa commodity.

Mundlack e Larson (1992) propuseram um modelo teórico que utiliza como base a lei do preço único e analisa como variações nos preços internacionais influenciam variações nos preços domésticos de determinada commodity agrícola. Segundo esse modelo, o preço doméstico de um produto agrícola é função do preço internacional do produto e da taxa de câmbio nominal, podendo ser algebricamente representado por

$$
P D_{i t}=P I_{t} \times T C_{t}
$$

em que $P D_{i t}$ é o preço doméstico da soja em grão no estado $i$, no período t; $P I_{t}$, o preço internacional da soja em grão no período $t$; e $T C_{t}$, a taxa de câmbio nominal no período $t$.

Na forma logarítmica, a equação 1 é reescrita da seguinte forma:

$$
p d_{i t}=p i_{t}+t c_{t}+u_{i t}
$$

em que o termo de erro $u_{i t}$ capta possíveis efeitos omitidos em razão da ausência de variáveis no modelo, como as políticas comerciais, que não serão consideradas diretamente neste trabalho. Assume-se que o termo de erro não se correlaciona com nenhuma das variáveis de entrada do modelo. 
Com isso, pelo modelo acima, os preços domésticos em moeda nacional da soja em grão são função do seu respectivo preço internacional, da taxa de câmbio nominal e do termo de erro, o que permite estimar o modelo a seguir:

$$
p d_{i t}=\alpha+\beta p i_{t}+\gamma t c_{t}+\varepsilon_{i t}
$$

no qual, $\alpha$ é o intercepto; $\beta$, a elasticidade do preço doméstico da soja em grão em reais em relação ao preço internacional (elasticidade de transmissão de preço); e $\gamma$, a elasticidade da taxa de câmbio nominal. Se os coeficientes referentes ao preço internacional e à taxa de câmbio forem próximos de um, uma mudança em uma dessas variáveis será repassada integralmente para o preço doméstico.

\subsection{Referencial Analítico}

Os estudos de transmissão de preços e integração regional fazem parte da análise de séries temporais e são comuns na literatura de economia agrícola brasileira (Margarido et al., 2007; Margarido, Turolla e Bueno, 2007; Cunha et al., 2008; Mendonça et al., 2011). O procedimento analítico do estudo da transmissão de preços neste trabalho é descrito a seguir.

Para alcançar os objetivos propostos neste trabalho, foram utilizados o modelo de correção de erro vetorial (VEC), que é uma versão reparametrizada do modelo de autorregressão vetorial (VAR) ${ }^{5}$, e o Teste de Causalidade de Granger, visando a verificar se valores passados de determinada variável contribuem para a previsão do comportamento futuro de outra variável.

Para execução de tais procedimentos, foi feita uma análise inicial das séries temporais, buscando identificar sua estacionariedade por meio do teste Dikey-Fuller aumentado (Augmented Dickey-Fuller - ADF),

5 Mais detalhes dos modelos VAR e VEC podem ser obtidos em Enders (1995, p. 294-304) e Lütkepohl (2004, capítulo 3, p. 86-158). 
seguindo os procedimentos propostos por Enders (1995), uma vez que é preciso que todas as séries sejam não estacionárias em nível e integradas de mesma ordem para a estimação do modelo VEC. Contudo, essa é uma condição necessária, mas não suficiente para que se tenha uma relação de curto prazo, ou transitória, e de longo prazo, ou permanente, entre as variáveis analisadas. Além disso, o Teste de Causalidade de Granger também deve ser feito a partir de variáveis estacionárias a fim de evitar resultados espúrios.

No VEC, as variáveis utilizadas são consideradas endógenas e determinadas de forma dinâmica por meio dos valores defasados de todas as variáveis (ENDERS, 1995). O modelo VEC com $k$ variáveis $\mathrm{e}^{n}$ defasagens pode ser representado pela seguinte forma matricial:

$$
\Delta P_{t}=\Gamma_{1} \Delta P_{t-1}+\cdots+\Gamma_{\mathrm{n}-1} \Delta P_{t-(n-1)}+\alpha \beta^{\prime} P_{\mathrm{t}-1}+\varepsilon_{\mathrm{t}}
$$

em que $\Delta P_{t}$ é um vetor $(\mathrm{kx} 1)$ dos preços da soja nos estados, mercado internacional e da taxa de câmbio em primeira diferença; $\Delta P_{t-j}, \mathrm{j}$ $=1,2, \ldots, \mathrm{n}$, são vetores $(\mathrm{kx} 1)$ dessas variáveis defasados em primeira diferença; $\Gamma_{\mathrm{i}}$ são os coeficientes de ajustamento transitório entre os preços; $\beta^{\prime} \mathbf{P}_{\mathrm{t}-1}$ são as " $\mathbf{r}$ " relações de longo prazo entre as variáveis, em que $\beta^{\prime}$ é uma matriz (rxk); a matriz $\alpha$ (kxr) representa os coeficientes de ajustamento de equilíbrio de longo prazo, ou de correção de erros; e $\varepsilon_{\mathrm{t}}$ é um vetor ( $\left.\mathrm{kx} 1\right)$ de erros aleatórios. As variáveis com o operador de diferença $(\boldsymbol{\Delta})$ são estacionárias e as $\mathbf{P}_{\mathrm{t}-1}$ são não estacionárias.

Contudo, para que se possa definir o modelo VEC na equação (4), o ranque da matriz $\alpha \beta^{\prime}$ (kxk) deve ser maior que zero e menor que k, ou seja, $0<\mathrm{r}<\mathrm{k}$. O ranque da matriz $\alpha \beta^{\prime}$ está associado diretamente ao número de raízes características dessa matriz. Assim, o número de raízes características não nulas da matriz $\alpha \beta^{\prime}$ é igual ao número de vetores de 
cointegração do modelo VEC. Para verificar o número de vetores de cointegração, utilizou-se o procedimento proposto por Johansen (1988), que consiste nos testes de razão de verossimilhança traço ( $\left.\lambda_{\text {trace }}\right)$ e máximo autovalor $\left(\lambda_{\max }\right)$. O primeiro procedimento testa a hipótese nula $\mathbf{r} \leq \mathbf{r}_{0}$ contra a hipótese alternativa de $\mathbf{r}>\mathbf{r}_{0}$. Por sua vez, a hipótese nula do segundo teste é que há $r$ vetores de cointegração, enquanto a alternativa é que há $(\mathbf{r}+\mathbf{1})$ vetores.

A análise da decomposição dos erros de previsão constituiu-se na última etapa do procedimento analítico após estimação do VEC. Segundo Margarido (2000), citado por Margarido et al. (2007), esse instrumental mostra o comportamento dinâmico apresentado pelas variáveis econômicas, permitindo separar a variância do erro de previsão para cada variável em componentes, que podem ser atribuídos pelas outras variáveis endógenas isoladamente, ou seja, revela, percentualmente, que efeito um choque imprevisto sobre determinada variável tem sobre as demais variáveis pertencentes ao sistema.

\subsection{Fonte de Dados}

A taxa de câmbio nominal (TC) real (R \$)/dólar estadunidense (US\$) foi retirada da base de dados do Instituto de Pesquisa Econômica Aplicada (IPEA, 2013). Os preços em $\mathrm{R} \$ / \mathrm{kg}$ pagos aos produtores de soja nos estados de Mato Grosso (PMT), Paraná (PPR), Rio Grande do Sul (PRS) e Goiás (PGO) e o preço internacional da soja (PI) em US\$/kg na Chicago Board of Trade (CBOT) foram obtidos da base de dados estatísticos da Associação Brasileira das Indústrias de Óleos Vegetais (ABIOVE, 2013). Todas as séries são médias mensais referentes ao período de janeiro de 1996 a dezembro de 2011.

\section{Resultados e Discussão}


A análise da estacionariedade pelo teste ADF indicou que todas as séries foram não estacionárias a 5\% de significância, mas estacionárias em primeira diferença a 1\% de significância, Tabela 1, atendendo, portanto, à condição necessária para a análise de cointegração ${ }^{6}$.

Tabela 1 - Testes ADF de raiz unitária para os logaritmos do preço internacional, da taxa de câmbio e dos preços domésticos, de 1996 a 2011.

\begin{tabular}{llccccc}
\hline \multirow{2}{*}{ Série } & \multicolumn{1}{c}{ Equação de teste } & Defasagens & $\begin{array}{c}\text { Estat. } \\
\text { de teste }\end{array}$ & Prob. & Valor 5\% & Crítico 1\% \\
\hline PI & constante e tendência & 1 & $-2,69067$ & 0,2417 & $-3,43365$ & $-4,007084$ \\
$\Delta$ PI & sem const. e sem tend. & 0 & $-9,59525$ & 0,0000 & $-1,94251$ & $-2,57719$ \\
\hline TC & constante e sem tendência & 1 & $-2,10071$ & 0,2447 & $-2,8766$ & $-3,464827$ \\
$\Delta$ TC & sem const. e sem tend. & 0 & $-8,71332$ & 0,0000 & $-1,94251$ & $-2,57719$ \\
\hline PMT & constante e tendência & 1 & $-2,34275$ & 0,4085 & $-3,43365$ & $-4,007084$ \\
$\Delta$ PMT & sem const. e sem tend. & 0 & $-10,3196$ & 0,0000 & $-1,94251$ & $-2,57719$ \\
\hline PPR & constante e tendência & 1 & $-2,52609$ & 0,3153 & $-3,43365$ & $-4,007084$ \\
$\Delta$ PPR & sem const. e sem tend. & 0 & $-8,82055$ & 0,000 & $-1,94251$ & $-2,57719$ \\
\hline PRS & constante e tendência & 1 & $-2,55722$ & 0,3006 & $-3,43365$ & $-4,007084$ \\
$\Delta$ PRS & sem const. e sem tend. & 0 & $-10,1953$ & 0,0000 & $-1,94251$ & $-2,57719$ \\
\hline PGO & sem const. e sem tend. & 0 & $-1,76517$ & 0,0737 & $-1,9425$ & $-2,577125$ \\
$\Delta$ PGO & sem const. e sem tend. & 0 & $-12,4223$ & 0,0000 & $-1,94251$ & $-2,57719$ \\
\hline
\end{tabular}

Notas: Foi utilizado o número de defasagens que minimizou o critério de Schwarz; rejeita-se a presença de raiz unitária ao nível de $1 \%$.

Os testes de causalidade de Granger efetuados, Tabela 2, apontaram que variações no preço internacional da soja e na taxa de câmbio afetaram os preços da soja pagos aos produtores nos quatro estados, conforme era esperado. A taxa de câmbio, por sua vez, não foi afetada por nenhuma das variáveis, enquanto o preço internacional foi influenciado por variações

6 Para a especificação da equação de teste, foram utilizados os procedimentos propostos por Enders (1995), páginas 221 a 238 e página 257. 
no preço em Mato Grosso a 10\% de significância. Apesar de não ser esperado, esse resultado pode estar relacionado ao fato de Mato Grosso ser o maior produtor nacional de soja. Corroborando esse raciocínio, Mato Grosso foi o único estado cujo preço não sofreu influência dos preços dos outros estados. O preço em Goiás foi afetado pelo preço em Mato Grosso e no Rio Grande do Sul, enquanto os preços recebidos no Paraná e no Rio Grande do Sul foram mutuamente afetados.

Tabela 2 - Resultados dos testes de causalidade de Granger ${ }^{7}$ para as variáveis analisadas

\begin{tabular}{ccccccc}
\hline & \multicolumn{5}{c}{ Variáveis Dependentes } \\
\cline { 2 - 6 } & PGO & PMT & PPR & PRS & PIN & TC \\
\cline { 2 - 6 } & \multicolumn{5}{c}{ Estatística $\chi^{2}$} \\
\hline PGO & - & $0,31^{\mathrm{NS}}$ & $1,28^{\mathrm{NS}}$ & $1,73^{\mathrm{NS}}$ & $0,17^{\mathrm{NS}}$ & $4,38^{\mathrm{NS}}$ \\
PMT & $12,27^{* * *}$ & - & $3,32^{\mathrm{NS}}$ & $1,22^{\mathrm{NS}}$ & $5,24^{*}$ & $4,35^{\mathrm{NS}}$ \\
PPR & $2,16^{\mathrm{NS}}$ & $0,90^{\mathrm{NS}}$ & - & $9,23^{* * *}$ & $0,84^{\mathrm{NS}}$ & $1,35^{\mathrm{NS}}$ \\
PRS & $7,95^{* *}$ & $2,51^{\mathrm{NS}}$ & $9,45^{* * *}$ & - & $1,31^{\mathrm{NS}}$ & $1,84^{\mathrm{NS}}$ \\
PI & $13,74^{*} * *$ & $5,14^{*}$ & $7,89^{* *}$ & $13,36^{* * *}$ & - & $0,11^{\mathrm{NS}}$ \\
TC & $13,93^{*} * *$ & $8,75^{* *}$ & $8,17^{* *}$ & $12,84^{* * *}$ & $0,16^{\mathrm{NS}}$ & - \\
\hline
\end{tabular}

*** Significativo a $1 \% ; * *$ Significativo a $5 \%$; * Significativo a $10 \%$; NS não significativo a $10 \%$.

Os testes de cointegração indicaram a 5\% de significância presença de quatro vetores de cointegração no conjunto de dados analisados (Tabela 3). Logo, foi possível estabelecer relações de longo prazo entre os preços da soja pagos aos produtores nos quatro estados analisados, o preço internacional em conjunto e a taxa de câmbio. Como o número de vetores de cointegração foi menor que o número de variáveis (rank reduzido), procedeu-se à estimação do modelo VEC.

7 O critério utilizado para definição do número de defasagens foi o de Shwarsz, contudo foi observada autocorrelação pelo teste de multiplicador de Lagrange a $1 \%$ para a oitava defasagem. Isso foi corrigido com a adição de mais uma defasagem (2). 
Tabela 3 - Testes de cointegração do traço e do máximo autovalor para as séries utilizadas

\begin{tabular}{ccccccc}
\hline $\begin{array}{c}\text { Hipótese } \\
\text { nula (Ho) }\end{array}$ & $\begin{array}{c}\text { Estatística } \\
\text { Traço }\end{array}$ & $\begin{array}{c}\text { Valor } \\
\text { crítico } \\
\mathbf{( 5 \% )}\end{array}$ & Prob. & $\begin{array}{c}\text { Estat. Máxi- } \\
\text { mo Eigen- } \\
\text { value }\end{array}$ & $\begin{array}{c}\text { Valor } \\
\text { crítico } \\
\mathbf{( 5 \% )}\end{array}$ & Prob. \\
\hline $\mathrm{r}=0$ & 188,9373 & 95,7537 & 0,0000 & 71,1951 & 40,0776 & 0,0000 \\
$\mathrm{r}=1$ & 117,7421 & 69,8189 & 0,0000 & 53,1382 & 33,8769 & 0,0001 \\
$\mathrm{r}=2$ & 64,6040 & 47,8561 & 0,0006 & 34,2137 & 27,5843 & 0,0061 \\
$\mathrm{r}=3$ & 30,3903 & 29,7971 & 0,0427 & 21,6501 & 21,1316 & 0,0423 \\
$\mathrm{r}=4$ & 8,7402 & 15,4947 & 0,3900 & 6,8246 & 14,2646 & 0,5101 \\
$\mathrm{r}=5$ & 1,9156 & 3,8415 & 0,1663 & 1,9156 & 3,8415 & 0,1663 \\
\hline
\end{tabular}

A dinâmica entre os preços da soja pagos aos produtores nos quatro estados analisados, o preço internacional da soja e a taxa de câmbio foram obtidos por meio do modelo VEC, cujos coeficientes de elasticidade de transmissão foram significativos ao nível de 1\% (Tabela 4). Assim, para o estado de Goiás, por exemplo, uma variação de 1\% no preço internacional levaria a uma variação de $1,05 \%$ no preço pago ao produtor no longo prazo. Para Mato Grosso, uma variação de $1 \%$ na taxa de câmbio levaria a uma variação de $1,02 \%$ no preço da soja pago ao produtor nesse estado, no longo prazo. Vale ressaltar que Mato Grosso foi o estado que apresentou as maiores elasticidades. 
Tabela 4 - Relações de longo prazo entre os preços pagos aos produtores brasileiros de soja com o preço internacional e a taxa de câmbio ${ }^{8}$

\begin{tabular}{lllll}
\hline & PMT & PPR & PRS & PGO \\
\hline PI & $-1,128749$ & $-1,055514$ & $-1,03634$ & $-1,0501$ \\
& {$[-22,9718]$} & {$[-26,4311]$} & {$[-23,7369]$} & {$[-31,3622]$} \\
TC & $-1,026449$ & $-0,995656$ & $-0,96265$ & $-1,016024$ \\
& {$[-20,7036]$} & {$[-24,7101]$} & {$[-21,8526]$} & {$[-30,0741]$} \\
\hline C & 0,084547 & 0,049074 & 0,051673 & 0,146052 \\
\hline
\end{tabular}

Nota: Os valores entre colchetes referem-se às estatísticas do teste t-student (1\%: 2,$57 ; 5 \%: 1,96 ; 10 \%: 1,64)$.

Alguns padrões puderam ser identificados pelas elasticidades de transmissão do preço internacional e pela taxa de câmbio para os preços pagos aos produtores brasileiros de soja. Primeiramente, ambas as elasticidades se situaram próximas a um para os quatro estados analisados, indicando que variações no mercado internacional de soja e no mercado de câmbio são repassadas integralmente ao preço pago ao produtor no longo prazo e que os estados analisados seguem um padrão comum de transmissão. Segundo Mendonça et al. (2011), coeficientes com valores acima de um são devidos à presença de custos de transação elevados e foram encontrados também em outros trabalhos (GONZÁLEZ-RIVERA; HELFAND, 2001; NOGUEIRA; AGUIAR; LIMA, 2005).

Vale destacar ainda o fato de as elasticidades relacionadas ao preço internacional serem maiores que as relacionadas à taxa de câmbio, indicando que o preço pago ao produtor brasileiro é mais influenciado pelo preço internacional do que pela taxa de câmbio. Margarido et al. (2007) chegaram a resultado semelhante ao verificarem que variações de preços no mercado internacional do trigo têm impacto mais acentuado

8 O VEC foi estimado com zero defasagem nas relações de curto prazo, como indicado pelo critério de Schwarz. Contudo, foi verificada autocorrelação pelo teste de multiplicador de Lagrange aplicado nos resíduos das estimações até a sexta defasagem. Para corrigir tal problema, foram adicionadas duas defasagens às equações de curto prazo. Pelo fato de a introdução de defasagens na relação de curto prazo ser meramente um procedimento para calcular erros padrão eficientes, os coeficientes de curto prazo não serão apresentados neste trabalho. 
sobre o preço da farinha na cidade de São Paulo do que variações na taxa de câmbio.

Os coeficientes de ajustamento à relação de longo prazo, Tabela 5, indicam o percentual do desequilíbrio de longo prazo corrigido por período, ou seja, a velocidade de ajustamento das variáveis em direção ao equilíbrio de longo prazo. O coeficiente de ajustamento da equação de Mato Grosso referente a desequilíbrios em seu próprio mercado em relação ao preço internacional e à taxa de câmbio, -0,1949, indica que $19,49 \%$ do desequilíbrio de longo prazo é corrigido por período. Desequilíbrios no mercado do Paraná são corrigidos por Mato Grosso à taxa de 20,21\% por período. Os coeficientes de ajuste na equação do Paraná respondem a desvios da relação de longo prazo nos estados de Goiás e Mato Grosso; na equação do Rio Grande do Sul, a desvios no próprio mercado, em Mato Grosso e em Goiás; e na equação de Goiás, aos próprios desvios e aos desvios em Mato Grosso.

Tabela 5 - Coeficientes de ajustamento à relação de longo prazo para os preços pagos aos produtores brasileiros de soja

\begin{tabular}{lcccc} 
Correção de erros & PMT & PPR & PRS & PGO \\
\hline PMT, PI e TC & $-0,194925$ & 0,12279 & 0,242547 & 0,195161 \\
& {$[-2,60155]$} & {$[1,84061]$} & {$[3,13756]$} & {$[2,69745]$} \\
PPR, PI e TC & 0,202073 & $-0,120401$ & 0,090923 & 0,101605 \\
& {$[1,93438]$} & {$[-1,29449]$} & {$[0,84360]$} & {$[1,00726]$} \\
PRS, PI e TC & $-0,057648$ & 0,046281 & $-0,304411$ & $-0,085495$ \\
& {$[-0,58669]$} & {$[0,52900]$} & {$[-3,00270]$} & {$[-0,90107]$} \\
PGO, PI e TC & $-0,073941$ & $-0,148789$ & $-0,2554$ & $-0,416562$ \\
& {$[-0,74123]$} & {$[-1,67523]$} & {$[-2,48154]$} & {$[-4,32458]$} \\
\hline
\end{tabular}

Nota: Os valores entre colchetes referem-se às estatísticas do teste t-student (1\%: 2,$57 ; 5 \%: 1,96 ; 10 \%: 1,64)$ 
Esses resultados indicam que os desvios da relação de longo prazo entre o preço pago ao produtor de soja em Mato Grosso, o preço internacional e a taxa de câmbio afetaram a dinâmica do preço de todos os estados analisados, evidenciando o papel desse estado na formação do preço da soja no Brasil, uma vez que, no curto prazo, os outros mercados seguiram a relação de preço de Mato Grosso com o preço internacional e a taxa de câmbio. Com relação à intensidade, os preços nos estados de Goiás e Rio Grande do Sul se ajustaram mais rapidamente aos desvios nas respectivas relações de longo prazo, uma vez que os maiores coeficientes de ajuste da relação de longo prazo foram verificados para esses estados. No caso de Mato Grosso e Paraná, a maior intensidade de ajuste foi verificada para a relação de longo prazo do Paraná e Goiás, respectivamente.

A análise do comportamento dinâmico das variáveis consideradas, efetuada pela decomposição da variância dos erros de previsão, em resposta a choques não antecipados numa das variáveis, foi limitada ao período de dois anos (Tabela 6). De maneira geral, os resultados evidenciaram o peso do preço internacional e da taxa de câmbio sobre o comportamento dos preços estaduais. 
Tabela 6 - Resultados da decomposição da variância dos erros de previsão em porcentagem

\begin{tabular}{lccccccc}
\hline \multirow{2}{*}{ Variável } & Período & & & & & & \\
& & LPMT & LPPR & LPRS & LPGO & LPIN & LTC \\
& (meses) & & & & & & \\
\hline LPMT & 1 & 82,88003 & 0,000000 & 0,000000 & 0,000000 & 12,08985 & 5,030115 \\
& 6 & 32,19529 & 5,913112 & 0,174354 & 1,237835 & 38,36260 & 22,11681 \\
& 12 & 19,83306 & 9,385947 & 1,080617 & 2,460452 & 40,48868 & 26,75125 \\
& 18 & 16,97982 & 12,81116 & 1,296984 & 3,334225 & 38,34916 & 27,22864 \\
& 24 & 15,56757 & 14,07813 & 1,245246 & 3,988283 & 37,23318 & 27,88759 \\
\hline LPPR & 1 & 8,190974 & 55,21285 & 0,000000 & 0,000000 & 23,74978 & 12,84639 \\
& 6 & 8,838834 & 21,33364 & 0,354573 & 2,651128 & 35,05691 & 31,76491 \\
& 12 & 10,40841 & 15,42119 & 0,723283 & 3,829566 & 36,40946 & 33,20809 \\
& 18 & 11,03735 & 15,75604 & 0,977749 & 4,343032 & 35,43193 & 32,45390 \\
& 24 & 11,23412 & 15,91536 & 1,009269 & 4,712871 & 34,79527 & 32,33311 \\
\hline LPRS & 1 & 6,958769 & 16,97361 & 40,85683 & 0,000000 & 23,05835 & 12,15244 \\
& 6 & 8,388633 & 9,421281 & 6,029864 & 3,142113 & 38,31142 & 34,70669 \\
& 12 & 10,45259 & 8,789751 & 3,676999 & 4,015656 & 39,09681 & 33,96820 \\
& 18 & 11,05299 & 11,74105 & 2,989887 & 4,444019 & 37,28977 & 32,48229 \\
& 24 & 11,23373 & 13,01811 & 2,504221 & 4,798574 & 36,30911 & 32,13625 \\
\hline LPGO & 1 & 11,37865 & 14,65924 & 5,062193 & 44,18507 & 19,75484 & 4,960010 \\
& 6 & 12,96502 & 9,083313 & 1,130499 & 4,699420 & 43,33927 & 28,78248 \\
& 12 & 11,92291 & 9,598399 & 1,867537 & 3,066617 & 42,57856 & 30,96598 \\
& 18 & 11,85618 & 12,67189 & 1,921631 & 3,340860 & 39,20466 & 31,00478 \\
& 24 & 11,76588 & 13,66456 & 1,722871 & 3,826441 & 37,37522 & 31,64502 \\
\hline
\end{tabular}

Vinte e quatro meses após um choque não antecipado no preço da soja em Mato Grosso, 15,57\% da variância dos erros de previsão, ou seja, de seu comportamento, é atribuída a si própria. Os $84,43 \%$ restantes são atribuídos ao preço internacional $(37,23 \%)$, à taxa de câmbio $(27,89 \%)$, ao preço da soja no Paraná, (14,08\%), no Rio Grande do Sul $(1,24 \%)$ e em Goiás (3,99\%). 
No caso do preço no Paraná, decorridos vinte e quatro meses após um choque não previsto sobre essa variável, 15,91\% de seu comportamento é devido a si própria; 34,79\%, ao preço internacional; 32,33\%, à taxa de câmbio; $11,23 \%$, ao preço da soja em Mato Grosso; 4,71\%, ao preço em Goiás; e 1,01\%, ao preço no Rio Grande do Sul.

Comportamento semelhante foi verificado para o preço no Rio Grande do Sul, em que, dois anos após um choque inesperado, apenas 2,50\% da variância dos erros de previsão é atribuída a essa variável. O restante é atribuído às demais variáveis, com destaque para o preço internacional e a taxa de câmbio, que, juntas, respondem por $68,45 \%$ de seu comportamento.

Por fim, passados vinte e quatro meses após um choque não antecipado sobre o preço em Goiás, somente 3,83\% da variância dos erros de previsão dessa variável é atribuída a si própria, enquanto 37,38\%, 31,65\%, 13,66\%, $11,77 \%$ e $1,72 \%$ da decomposição da variância dos erros de previsão se devem, respectivamente, ao preço internacional, à taxa de câmbio, ao preço da soja no Paraná e no Rio Grande do Sul.

\section{Considerações finais}

A preocupação dos produtores de soja com variações na taxa de câmbio é confirmada neste estudo, uma vez que foi verificada estreita relação entre os preços pagos aos produtores e a taxa de câmbio. Entretanto, quando comparada com o preço internacional, a taxa de câmbio desempenha papel secundário, dado que as técnicas utilizadas mostraram que os preços domésticos são mais sensíveis a variações no mercado internacional do que na taxa de câmbio.

Os mercados analisados mostraram-se integrados, havendo relação de equilíbrio, no longo prazo, entre os preços recebidos pelos produtores dos quatro estados analisados. Verificou-se elevada interdependência dos preços da soja nos estados em relação ao preço em Mato Grosso, bem como ao preço internacional e à taxa de câmbio. Com isso, confirmou-se 
a relevância do maior estado brasileiro produtor de soja na formação dos preços nos principais mercados dessa commodity.

Os resultados deste trabalho permitem afirmar, para o complexo agroindustrial da soja, que tanto a taxa de câmbio quanto o preço internacional afetam o preço doméstico, o que ressalta a necessidade de esses agentes acompanharem não somente o mercado da soja, mas também o mercado cambial, e que mudanças cambiais promovem impactos aproximadamente iguais aos do preço internacional sobre o mercado interno, o que mostra a vulnerabilidade do setor à instabilidade cambial.

\section{Referências}

ASSOCIAÇÃO BRASILEIRA DAS INDÚSTRIAS DE ÓLEOS VEGETAIS - ABIOVE. Estatística. 2013. Disponível em: <http:// www.abiove.org.br/site/index.php?page $=$ estatistica\&area $=$ NC0yLTE $\equiv$. Acesso em: 23 ago. 2013.

BAFFES, J.; GARDNER, B. The transmission of world commodity prices to domestic markets under policy reforms in developing countries. The Journal of Policy Reform, v. 6, n.3, p. 159-180, 2003.

BRASIL (2013a). MINISTÉRIO DA GRICULTURA, PECUÁRIA E ABASTECIMENTO. Soja. Disponível em: <http://www.agricultura. gov.br/vegetal/culturas/soja>. Acesso em: 23 ago. 2013.

BRASIL (2013b). MINISTÉRIO DA GRICULTURA, PECUÁRIA E ABASTECIMENTO. AGROSTAT. Estatísticas de Comércio Exterior do Agronegócio Brasileiro. Disponível em: <www.agrostat.agricultura. gov.br>. Acesso em: 08 set. 2013.

CUNHA, D. A.; BRAGA, M. J.; CAMPOS, A. C.; FERNANDES, L. L. Transmissão de preços no mercado internacional de café robusta. Revista de Economia e Agronegócio, Viçosa, v.6, n. 1, p. 1-28, 2008. 
ENDERS, W. Applied econometric time series. Nova York: John Wiley \& Sons, Inc., 1995. 433 p.

FRAGA, G. J.; ARRUDA, C. S.; ALVES, A. F.; PARRÉ, J. L. Revista Análise Econômica, Porto Alegre, v. 26, n. 49, p. 193-210, mar. 2008.

GONZÁLEZ-RIVERA, G.; HELFAND, S. M. The extent, pattern and degree of market integration: a multivariate approach for the Brazilian rice market. American Journal of Agricultural Economics, v. 83, n. 3, p. 576-592, 2001.

INSTITUTO BRASILEIRO DE GEOGRAFIA E ESTATÍSTICA IBGE. Pesquisa agrícola municipal - 2011. Disponível em: $<\mathrm{http}: / /$ www.sidra.ibge.gov.br/bda/pesquisas/pam/default.asp?o $=18 \& \mathrm{i}=\mathrm{P}>$. Acesso em: 08 set. 2013.

KRUGMAN, P. R.; OBSTFELD, M.; Economia internacional: teoria e política. São Paulo: Pearson Addison Wesley, 2005.

LÜTKEPOHL, H. Vector autoregressive and vector error correction models. In: LÜTKEPOHL, H., KRÄTZIG, M. (Eds.). Applied time series econometrics. Cambridge, UK: Cambridge University Press. 2004. cap. 3, p. 86-158.

MARGARIDO, M. A. Transmissão de preços agrícolas internacionais sobre preços agrícolas domésticos: o caso do Brasil, 2000, 173 p. Tese (Doutorado em Economia Aplicada) - USP/Esalq, Piracicaba.

MARGARIDO, M. A.; BUENO, C. R. F.; MARTINS, V. A.; TOMAZ, I. F. Análise dos efeitos preço e câmbio sobre os preços da farinha de trigo na cidade de São Paulo: uma aplicação de modelos de séries de tempo. Pesquisa \& Debate, São Paulo, v. 18, n. 2, p. 251-281, 2007.

MARGARIDO, M. A.; FERNANDES, J. M.; TUROLLA, F. A. Agric., São Paulo, v. 47, n. 2, p. 71-85, 2002. 
MARGARIDO, M. A.; TUROLLA, F. A.; BUENO, C. R. F. The world market for soybeans: price transmission into Brazil and effects from the timing of crop and trade. Nova Economia, Belo Horizonte, v. 17, n. 2, p. 241-270, 2007.

MENDONÇA, T. G.; LIMA, J. E.; LÍRIO, V. S.; PEREIRA, V. F. Integração espacial no mercado brasileiro de soja em grão, no período de 1994 a 2008. Análise Econômica, Porto Alegre, v. 29, n. 55, p. 235258, mar. 2011.

MUNDLACK, Y.; LARSON, D. F. On the transmission of world agricultural prices. The World Bank Economic Review, v. 6, n. 1, p. 399-422, 1992.

NOGUEIRA, F. T. P.; AGUIAR, D. R. D.; LIMA, J. E. Integração espacial no mercado brasileiro de café arábica. Nova Economia, v. 15, n. 2, p. 91-112, 2005. 
REVISTA DE ECONOMIA E AGRONEGÓCIO, VOL.11, $N^{\circ} 3$ 